\title{
Beiträge zur Kenntnis der Enzymwirkung (Protease, Arginase und Asparaginase) und der Analyse der menschlichen . Geschwülste (Hypernephrom, Gallertkrebs und Sarkom).
}

\author{
Von \\ Tamezi Hirooka, Daizi Hiwatasi, Tooru Sakai, Kooiti Dei

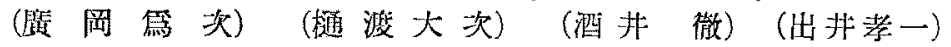 \\ und Tadayosi Sugawara. \\ (菅 原 忠 義) \\ (Aus dem Medizinisch-chemischen Institut der Medizinischen \\ - Fakultät der Kaiserlichen Tohoku-Universität, Sendai. \\ Direktor: Prof.Dr.S. Utzino.)
}

Auf dem umfangreichen Gebiet der Tumorforschungen ist der Aufgabe der Tumorenzymologie eine neue wichtige Richtung gewiesen worden. In dieser Hinsicht bleibt aber wegen des Materialsmangels an menschlichen Geschwülsten noch viel zu untersuchen übrig. In vorliegender Arbeit kamen in der Hauptsache die Protease, Arginase und Asparaginase des Hypernephroms und die Protease des Magenkrebses und des Sarkoms in Betracht. Nach der Beobachtung über die Proteasenwirkung des Maus- und des Kaninchenkarzinoms haben Utzino und Yoshio ka $(1939)^{12)}$ eine deutliche Kathepsin wirkung der Magenkrebsmazeration bei $\mathrm{pH} 4,0-6,0$ festgestellt, die durch Cysteinzusatz aktiviert werden konnte, während die proteolytische Wirkung auf Casein oder Gelatine bei $\mathrm{pH}$ 2,0 sich als sehr unbedeutend erwies, und auch auf die leichte Spaltung des Di- oder Tripeptids wie auch des Peptons bei $\mathrm{pH}$ 8,0 hingewiesen. Aus diesen Angaben scheint hier in Magenkrebsmazeration die katheptische wie ereptische Wirkung in hervorragenderem Grade als die Pepsinwirkung aufzutreten und es hat den Anschein, als ob das Proteasensystem des Magenkrebses nur wenig mit dem Pepsinenzym zu tun hat.

Was nun den Zusammenhang der Hypernephromproteolyse mit der Nierenproteolyse anbelangt, so wissen wir noch weniger. Nachdem Edlbacher et al. (1927) ${ }^{3)}$ darauf hingewiesen hatten, dass alle 
bösartigen Menschen- wie Säugertumoren, ohne Unterschied ob Krebs oder Sarkom, sich durch starke Arginasewirkung auszeichnen, beziehen sich die Untersuchungen von Wald schmidt-Leitz et al. (1933)", Edlbacher et al. $(1934)^{5}$, Minato $(1934)^{6)}$ und auch von Kaiju $(1938)^{7}$ in der Hauptsache auf die Arginasewirkung des Tier- und Hühnersarkoms unter Berücksichtigung des Zeitverlaufs nach der Impfung bzw. der Alterung.

Nach der Angabe von Kaiju (1938) ${ }^{7)}$ war die Arginasewirkung des Mazerationssaftes des Magenkarzinoms von Menschen viel stärker als die des nichtkarzinomatösen Umgebungsgewebes des Magenkarzinoms und die Arginaseaktivität scheint im innigen Zusammenhang mit den morphologischen Befunden der Zelltätigkeit zu stehen.

In vorliegender Arbeit wurde die Arginasewirkung des menschlichen Sarkomgewebes und des Hypernephroms neben dem Nierengewebe unter Berücksichtigung der Manganaktivierung studiert. Nach der Angabe von Waldschmidt-Leitz et al. $(1933)^{4)}$ zeigen Kathepsin, Phosphatase sowie die Adenylsäure- und Guanidindesamidase mit der Alterung der Tumoren (Sarkom Philadelphia 1 und Carcinom Walker 256), der Zunahme an nekrotischem Gewebe in Tumoren, eine stetige und bedeutende Abnahme der Enzymmenge, während die Arginasemenge mit steigender Nekrose erheblich zunimmt.

Unsere Ergebnisse sind kurz zusammengestellt wie folgt. Die genuinen Eiweisskörper wie Casein und Mucin wurden durch die Mazeration des scheinbar intakten Magenschleimhautteils vom Karzinommagen deutlich bei $\mathrm{pH}$ 2,0 angegriffen und das Casein auch bei pH 5,0, nicht aber das Mucin (Tabelle I). Die Karzinommasse vermag die Proteinkörper bei $\mathrm{pH}$ 5,0-5,6 noch stärker abzuspalten, die oberflächliche Schicht derselben nur das Casein bei pH 2,0, nicht aber das Mucin (Tabelle I). Diesen Beobachtungen nach scheint die Proteinasewirkung von katheptischer Natur dem Magenkarzinom (Gallertkrebs) mehr zuteil zu werden, als die Pepsinwirkung, da eine schwache Spaltung des Caseins bei pH 2,0, nicht aber des Mucins, auch durch das Karzinomenzym von katheptischer Natur, das sich durch die optimale Wirkung bei $\mathrm{pH}$ 5,0-6,0 auszeichnen kann, wenn auch in schwachem Grad wie in diesem Beispiel hervorgerufen werden mag. Im Gegensatz zu diesen Ergebnissen trat die Proteolyse bei $\mathrm{pH}$ 2,0 durch die scheinbar intakte umgebende Magenschleimhaut stark deutlich zutage, nicht aber bei pH 5,0, indem gegenüber der Enzym wirkung bei pH 5,0 das Mucin widerstand, das sicher durch den Hundemagensaft hydrolysierbar ist (Hirooka, noch nicht veröffentlicht). 
Was die Sarkomprotease betrifft, so trat die bei $\mathrm{pH}$ 7,5 wirkende Ereptasewirkung, wie Peptonase und Dipeptidase sehr auffallend in Erscheinung, und auch die Kathepsinwirkung fiel bei pH 4,5 positiv aus, indem der Cysteinzusatz fast ohne Einfluss auf das Sarkomkathepsin bleibt, und zwar scheint er etwas hemmend auf die Caseinhydrolyse einzuwirken (Tabelle II).

In einem Beispiel des Hypernephroms kam die Proteasewirkung desselben im Vergleich mit dem umgebenden Nierengewebe in Betracht. Die ereptische Peptonase-wie Katheptasewirkung (Kathepsin imengeren Sinne und katheptische Peptonase) der Nierenmazeration erwiesen sich sehr wirksam und die letztere wurde durch Cysteinzusatz deutlich aktiviert (Tabelle III). 'Die Ereptase wie Katheptase des Hypernephroms, das an demselben Nierengewebe angewachsen ist, fielen auch positiv aus, wenn auch viel schwächer als die des Nierengewebes, indem durch Cysteinzusatz nur die Gelatinehydrolyse beschleunigt wurde, nicht aber die Peptonhydrolyse (Tabelle IV). Für diese niedrigere Aktivität der Hypernephromproteolyse muss man wohl zuerst das Wassergehalt des Hypernephromgewebes berücksichtigen.

Die Arginaseaktivität des Menschensarkoms wie auch die des $\mathrm{Hy}-$ pernephroms steht im allgemeinen niedrig, jedoch wurde diejenige des Sarkoms durch Manganzusatz bedeutend aktiviert (Tabelle V u. VI). Die Vollaktivität des Hypernephroms erwies sich viel schwächer als diejenige des Nierengewebes. In Voraussetzung des Zusammenhangs der Arginaseaktivität mit der Zelltätigkeit, wie darauf schon von $\mathrm{K}$ aiju $(1938)^{7}$ ) beim Magenkarzinom hingewiesen wurde, kann man hier auch gut verstehen, dass das Sarkomgewebe im Gegensatz zu Hypernephrom ein sehr schnelles Wachstum zeigt.

Die Asparaginaseaktivität des Hypernephroms erwies sich viel schwächer als die des Nierengewebes (Tabelle VII).

Um einen analytisch-chemischen Einblick in das Tumorgewebe zu gewinnen, hat der eine von uns (Sugaw ara) hier die Stickstoffverteilung im Hydrolysat des wasserunlöslichen entfetteten Hypernephroms vergleichend mit dem umgebenden Nierengewebe studiert. In dieser Hinsicht haben Utzino und Mitarbeiter (Utzino u. Iida, 1935); Takamats u, 1935'); Utzino u. Shimazu, 1936 ${ }^{10}$ ) uber die Stickstoffverteilung im Hydrolysat des Tiersarkomgewebes berichtet. In den Ergebnissen betreffs der N-Verteilung im Kaninchensarkom (F ujinami-Kato's Stamm) fanden Utzino und Shimazu (1936) ${ }^{10}$ keinen besonderen Unterschied zwischen dem Sarkomgewebe und den anderen Normalgewebe, wie Leber, Niere, Lunge, Muskel oder Herz- 
muskel. Das Sarkoingewebe scheint nur einen etwas grössèren Wert des Argininfraktion-N (13\%) zu zeigen, indem es 60\% Monoaminosäure-N, $3 \%$ Histidin-N oder $9 \%$ Lysin-N enthält. Die hier erzielten Ergebnisse zeigen, dass man in der Stickstoffverteilung im untersuchten Gewebe fast keinen besonderen Unterschied zwischen Nierengewebe und Hypernephrom feststellen konnte, das 12\% Arginin-N, $3 \%$ Histidin- $\mathrm{N}$ und 4,6\% Lysin-N zeigte, wobei letzterer etwas kleiner als derjenige des Nierengewebes $(6 \%)$ zu sein scheint.

\section{Zusammenfassung.}

1) In der Magenkrebsmazeration (Gallertkrebs) befindet sich die Proteinasewirkung, die Casein wie Mucin bedeutend bei $\mathrm{pH} 5,0-5,6$ anzugreifen, jedoch bei pH 2,0 nicht Mucin sondern nur Casein zu hydrolysieren vermag, während diese beiden Eiweisskörper durch die Mazeration der scheinbar intakten Umgebungsschleimhaut des Magenkrebes bei $\mathrm{pH}$ 2,0 hydrolysierbar sind, bei $\mathrm{pH}$ 5,0 aber nicht Mucin sondern Casein. Es hat den Anschein, als ob im Magenkrebsgewebe die katheptische Wirkung erheblicher zutage trat.

2) Im Sarkomgewebe (Spindelzellensarkom) wurde die deutliche Ereptasewirkung (Peptonase und Dipeptidase) festgestellt; die Kathepsinwirkung fiel auch positiv aus.

3) Im Hypernephrom wurde auch die Ereptase (Peptonase) und Katheptase (Kathepsin und katheptische Peptonase) in noch schwächerem Grad, als im umgebenden Nierengewebe bestätigt.

4) Die Arginaseaktivität des Sarkomgewebes wurde durch Manganzusatz ziemlich verstärkt; diejenige des Hypernephroms scheint aber trotz der Manganaktivierung viel schwächer als die des Nierengewebes zu sein, ebenso ist die Asparaginase des Hypernephroms schwächer.

5) In der Stickstoffverteilung im wasserunlöslichen entfetteten Gewebe des Hypernephroms und des intakten Nierenteils fanden wir fast keinen besonderen Unterschied, indem die Analysenwerte 12\% Arginin-N, 3\% Histidin- $\mathrm{N}$ und 4,6\% Lysin-N betragen.

Diese Arbeit wurde mit Unterstützung der Sa i to o-Hoonkai ausgeführt, der wir an dieser Stelle unseren verbindlichsten Dank aussprechen möchte.

\section{Literatur.}

(1) Utzino, S. u. M. Yoshioka(1939): Kagaku-Kenkyusho Kooen-shuu, 10, 148 u. 153.

(2) Utzino, S. u. M. Yoshioka (1939): ibid,, 10, 157. 
(3) Edlbacher, S. u. K.W. Merz (1937): Zeits. physiol. Chem., 171, 252.

(4) Waldschmidt-Leitz, E., E. Mc Don ald et al. (1933): ibid., $219,115$.

(5) Edlbacher, S. a. F. Koller (1934): ibid., 227, 99.

(6) Minato, I. (1934): Osaka-Igakkai Zassi, 33, 283.

(7) Kaiju, M. (1938): Journ. Biochem., 27, 35.

(8) Utzino, S. u. T. Iida (1935): Kagaku-Kenkyusho Kooen-shuu, 6, 78.

(9) T a ka mats u, M. (1935): Kyoto Igakkai Zassi, 32, 585.

(10) Utzino, S. u. K. Shim a u (1936): Kagaku-Kenkyusho Kooen-shnu, 7, 131.

(11) Tom ot a, S. (1940): Journ. Biochem., 32, 307.

(12) Hiwatasi, D. (1941): Dieses Journ., 41, 295.

(13) Chen, T.-T. (1939): ibid., 37, 367.

\section{Enzymologischer Teil.}

\section{Tabelle I.}

\section{Proteasewirkung des Magentarzinoms.}

Enzym: Als Enzymmaterial steht uns die chirurgisch operierte Magenkrebsmasse*) mit umgebendem Magenteil zur Verfügung, die makroskopisch in einen Krebsteil (Gallertkrebs) nnd in einen scheinbar gesunden Magenschleimhautteil getrennt und auf die Proteinaseaktivität bei $\mathrm{pH} 2,0$ und bei $\mathrm{pH} 5,0$ hin geprüft wurde. Von dem karzinomatösen Teil wurde die oberflächliche Schicht vorsichtig abgetrennt und besonders auf ihre Proteaseaktivität bei $\mathrm{pH}$ 2,0 untersucht. Je ein Teil Gewebesbrei wurde mit der dreifachen Volummenge Glycerin-Wasser (1:1) gut zerrieben, durch Gaze koliert und versucht.

Versuchsansatz: $20 \mathrm{ccm}$ 1\%iger Substratpufferlösung (bei pH 2,0,5,0 oder $5,6)+2 \mathrm{ccm}$ Mazeration der Tumormasse, der Tumoroberflächenschicht oder der Magenschleimhaut+Toluol; digeriert bei $37^{\circ} \mathrm{C}$. Zur Kontrolle wurde die Enzym-Pufferlösung ohne Substrat digeriert. Nach einer gewissen Digestionszeit hat man die Acidität in $4 \mathrm{ccm}$ Digestat nach $\mathrm{S}$ örensen mit $0,1 \mathrm{n}-\mathrm{NaOH}$ formoltitriert. Nach dem Abzug der Kontrollwerte sind die korrigierten Werte der Aciditätszunahme des Hauptversuchs in Tabelle angegeben.

Aciditätszunahme in $4 \mathrm{ccm}$ Digestat ( $\mathrm{cem} 0,1 \mathrm{n}-\mathrm{NaOH}$ )

\begin{tabular}{|c|c|c|c|c|c|c|c|c|c|c|}
\hline \multirow[b]{3}{*}{$\begin{array}{l}\text { Zeit } \\
\text { (Std.) } \\
\end{array}$} & \multirow{2}{*}{\multicolumn{2}{|c|}{ 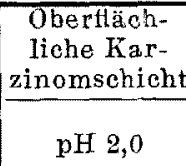 }} & \multicolumn{4}{|c|}{$\begin{array}{c}\text { Umgebende } \\
\text { Magenschleimhaut }\end{array}$} & \multicolumn{4}{|c|}{ Karzinommasse } \\
\hline & & & \multicolumn{2}{|c|}{$\mathrm{pH} 2,0$} & \multicolumn{2}{|c|}{ pH 5,0} & \multicolumn{2}{|c|}{ pH 5,0 } & \multicolumn{2}{|c|}{$\mathrm{pH} 5,6$} \\
\hline & Macin & Casein & Mucin & Casein & Mucin & Casein & Muein & Casein & Mucin & Casein \\
\hline 24 & 0,1 & 0,4 & 0,1 & 0 & $-0,1$ & 0 & 0,1 & 0,5 & 0,1 & 0,3 \\
\hline 72 & 0 & 0,4 & 0,3 & 0,4 & $-0,2$ & 0,4 & 0,1 & 0,6 & 0,2 & 0,7 \\
\hline 120 & 0 & 0,4 & 0,3 & 0,5 & $-0,1$ & 0,5 & 0,1 & 0,7 & 0,2 & 1,0 \\
\hline
\end{tabular}

*) Für die freundliche Überlassung des Geschwülstmaterials sind wir Herren Prof. Dr. Sh. Sugimura und Prof. Dr. Sh. Sekiguchi in den chirurgischen Kliniken zu grossem Danke verpflichtet. 


\section{Tabelle II. \\ Proteasewirkung des Menschensarkoms.}

Enzym: Die operierte Sarkommasse (Spindelzellensarkom) wurde fein zerschnitten, mit der 3fachen Volummenge Glycerin-Wasser (1:1) gut zerrieben und durch Gaze koliert. Die kolierte Mazeration gelangte zur Verwendung. Zur Aktivierung des Kathepsins wurden $2 \mathrm{ccm}$ Mazeration mit $2 \mathrm{ccm} \mathrm{0,2} \mathrm{Mol-}$ Cystein $\mathrm{HCl}$ (neutralisiert) bei $37^{\circ} \mathrm{C} 30 \mathrm{Min}$. lang behandelt. Zur Kontrolle kamen 2 ccm Wasser ohne Cystein zur Beobachtung.

Versuchsansatz für Ereptasewirkung: $5 \mathrm{ccm} \mathrm{0,2} \mathrm{Mol-Diglycinlösung} \mathrm{mit}$ $13 \mathrm{ccm}$ Phosphatpufferlösung ( $\mathrm{pH} 7,5)$ oder $18 \mathrm{~cm} 2 \%$ iger Peptonpufferlösung . $(\mathrm{pH} 7,5)+2 \mathrm{ecm}$ Sarkommazeration + Toluol.

Versuchsansatz für Kathepsinwirkung: $18 \mathrm{ccm} 2 \%$ iger Proteinpufferlösung $(\mathrm{pH} 4,5)+2 \mathrm{ccm}$ Sarkommazeration mit $2 \mathrm{ccm} 0,2$ Mol-Cystein HCl-Lösung für Aktivierung oder mit $2 \mathrm{ccm}$ Wasser ohne Cystein für Kontrolle + Toluol ; digeriert bei $37^{\circ} \mathrm{C}$. Kontrolle mit Enzym-Puffer ohne Substrat. Aciditätsbestimmung in 4 ccm Digestat mit $0,1 \mathrm{n}-\mathrm{NaOH}$ nach $\mathrm{S}$ örensen. Die korrigierten Werte der Aciditätszunahme angegeben.

Aciditätszunahme in $4 \mathrm{ccm}$ Digestat (ccm 0,1 n-NaOH)

\begin{tabular}{|c|c|c|c|c|c|c|}
\hline $\mathrm{pH}$ & \multicolumn{2}{|c|}{$\mathrm{pH} 7,5$} & \multicolumn{4}{|c|}{$\mathrm{pH} 4,5$} \\
\hline Sub- & \multirow{2}{*}{ Diglycin } & \multirow{2}{*}{$\begin{array}{l}\text { Pepton- } \\
\text { Witte }\end{array}$} & \multicolumn{2}{|c|}{ ohne Cystein } & \multicolumn{2}{|c|}{ mit Cystein } \\
\hline Zeit (Std.) & & & Casein & Gelatine & Casein & Gelatine \\
\hline 24 & 1,5 & 0,8 & 0,2 & 0 & $-0,1$ & 0,2 \\
\hline 72 & 1,6 & 0,8 & 0,3 & 0,2 & $-0,4$ & 0,1 \\
\hline 120 & - & - & 0,4 & 0,2 & $-0,4$ & 0,1 \\
\hline
\end{tabular}

\section{Tabelle Ш.}

Proteasewirkung des am Hypernephrom anliegenden Nierengewwebes.

Enzym: Das Nierengewebe, das am Hypernephrom anliegend bleibt und makroskopisch intakt zu sein schien, wurde mit der 3fachen Volummenge Glycerin-Wasser (1:1) wie in Tabelle II mazeriert, koliert und geprüft.

Versuchsansatz: $5 \mathrm{ccm} 8 \%$ iger Substratlösung $+11 \mathrm{ccm}$ Phosphatpuffer $+2 \mathrm{ccm}$ Gewebemazeration mit $2 \mathrm{ccm} 0,1$ Mol-Cystein oder mit $2 \mathrm{ccm}$ Wasser ohne Cystein + Toluol; bei $\mathrm{pH} 4,5$ und bei $37^{\circ} \mathrm{C}$ digeriert.

Für Ereptase bei pH 7,2 digeriert. Kontrolle wie in Tabelle II. Die korrigierten Werte angegeben. 
Aciditätszunahme in $4 \mathrm{ccm}$ Digestat $(\mathrm{ccm} 0,1 \mathrm{n}-\mathrm{NaOH})$

\begin{tabular}{|c|c|c|c|c|c|c|c|c|c|c|}
\hline \multirow{2}{*}{$\mathrm{pH}$} & \multirow{2}{*}{$\underbrace{\text { Aktivie- }}_{\text {Substrate }} \begin{array}{c}\text { Zeit } \\
\text { (Stang })\end{array}$} & \multicolumn{3}{|c|}{ a) ohne Cystein } & \multicolumn{3}{|c|}{ b) mit Cystein } & \multicolumn{3}{|c|}{$\begin{array}{c}\text { Zunahme durch } \\
\text { Aktivierung (b-a) }\end{array}$} \\
\hline & & 22 & 72 & 120 & 24 & 72 & 180 & 24 & 72 & 120 \\
\hline \multirow{2}{*}{4,5} & Gelatine C & 0,45 & 0,98 & 1,12 & 0,52 & 1,22 & 1,65 & 0,07 & 0,24 & 0,53 \\
\hline & Pepton K & 0,58 & 0,77 & 1,00 & 0,72 & 0,97 & 1,30 & 0,14 & 0,20 & 0,30 \\
\hline 7,2 & Pepton $\mathrm{K}$ & 0,88 & 1,10 & 1,08 & - & - & - & - & - & - \\
\hline
\end{tabular}

\section{Tabelle IV.}

Proteasewirkung des Hypernephroms.

Enzym: Das nephrektomierte Hypernephromgewebe wurde mit der 3fachen Volummenge Glycerin-Wasser $(1: 1)$ gut zerrieben, durch Gaze koliert und untersucht.

Versuchsansatz: $5 \mathrm{ccm} 8 \%$ Substratlösung $+11,0 \mathrm{ccm}$ Phosphatpuffer + $2 \mathrm{cem}$ Mazeration mit $2,0 \mathrm{ccm} 0,1 \mathrm{Mol}$-Cysteinlösung (bei $\mathrm{pH} 6,0$ und $37^{\circ} \mathrm{C} 30$ Min. aktiviert) oder $+2,0 \mathrm{ccm}$ Mazeration mit 2,0 ccm Wasser ohne Cystein (bei $\mathrm{pH} 6,0$ und $37^{\circ} \mathrm{C} 30 \mathrm{Min}$. aufbewahrt.) + Toluol; digeriert bei pH 4,5 und bei $37^{\circ} \mathrm{C}$.

Für die Ereptase-Probe wurde der Ansatz ohne Cystein bei pH 7,2 unter Toluol bei $37^{\circ} \mathrm{C}$ digeriert. Kontrolle mit Enzympuffer ohne Substrat wie Tabelle III.

Formoltitration in $4 \mathrm{ccm}$ Digestat. Die korrigierten Werte angegeben.

Aciditatszunahme in $4 \mathrm{ccm}$ Digestat ( $\mathrm{ccm} 0,1 \mathrm{n}-\mathrm{NaOH})$

\begin{tabular}{|c|c|c|c|c|c|c|c|c|c|c|}
\hline \multirow{2}{*}{$\dot{\mathbf{p}} \mathbf{H}$} & \multirow{2}{*}{$\underbrace{\text { Zeit }}_{\text {Substrate }}$ (Std.) } & \multicolumn{3}{|c|}{ a) ohne Cystein } & \multicolumn{3}{|c|}{ b) mit Cystein } & \multicolumn{3}{|c|}{$\begin{array}{c}\text { Zunahme durch } \\
\text { Aktivierung }(b-a)\end{array}$} \\
\hline & & 24 & 72 & 120 & 24 & 72 & 120 & 24 & 72 & 120 \\
\hline \multirow{2}{*}{4,5} & Gelatine C & 0,08 & 0,10 & 0,22 & 0,23 & 0,48 & 0,58 & 0,15 & 0,38 & 0,36 \\
\hline & Pepton K & 0,33 & 0,50 & 0,55 & 0,23 & 0,40 & 0,47 & $-0,10$ & $-0,10$ & $-0,08$ \\
\hline 7,2 & Pepton K & 0,53 & 0,83 & 0,77 & - & $\cdots$ & - & - & - & - \\
\hline
\end{tabular}

Tabelle V.

Arginasewirkung des Menschensarkoms.

Enzym: Ebenso wie in Tabelle II. Die Arginasebestimmung wurde in der 
Weise ausgeführt, wie sie in der Abhandlung von Tomota $(1940)^{11)}$ beschrieben ist.

Versuchsansatz: $3 \mathrm{ccm}$ Argininchlorhydratlösung (Titer $=28,8 \mathrm{ccm} 0,02$ $\left.\mathrm{n}-\mathrm{H}_{2} \mathrm{SO}_{4}\right)+2 \mathrm{ccm}$ Sarkommazeration mit $1 \mathrm{ccm} 3 / 400 \mathrm{Mol}-\mathrm{MnSO}_{4}$-Lösung oder $1 \mathrm{ccm}$ Wasser ohne $\mathrm{MnSO}_{*}+9 \mathrm{ccm}$ 0,1 Mol-Glycin-NaCl-NaOH-Puffer + Toluol; bei $37^{\circ} \mathrm{C} 24$ Stunden lang digeriert. Umpufferung $(\mathrm{pH} \mathrm{7,0)}$ und Ureasezerlegung ( 1 Stunde lang) und dann Ammoniakbestimmung nach Folin.

Kontrollversuche mit Sarkommazeration mit Puffer ohne Arginin. Nach Abzug der Kontrollwerte sind die korrigierten Werte des Säureverbrauchs zur Neutralisation des Ammoniaks in Tabelle angegeben.

Zunahme des Säureverbrauchs

\begin{tabular}{c|c|c}
\hline & ccm $0,02 \mathrm{n}-\mathrm{H}_{2} \mathrm{SO}_{4}$ & Spaltung \% \\
\hline ohne $\mathrm{MnSO}_{4}$ & 0,9 & 3,3 \\
mit $\mathrm{MnSO}_{4}$ & 16,6 & 59,9
\end{tabular}

Tabelle VI.

Arginasewirlung des Hypernephroms und des Nierengewebes des Menschen.

Enzym: Ebenso wie in Tabelle III und IV. Die Arginasebestimmung auch wie in Tabelle $V$.

Versuchsansatz: $1 \mathrm{ccm}$ Gewebemazeration mit $1 \mathrm{ccm}$ Wasser oder mit 1 ccm 0,005 Mol- $\mathrm{MnSO}_{4}$-Lösung $+4 \mathrm{ccm}$ Argininchlorhydratlösung (Titer 46,8 $\mathrm{ccm})+9 \mathrm{ccm} 0,1 \mathrm{Mol}-$ Glycin-NaCl-NaOH-Puffer $(\mathrm{pH} \mathrm{9,2)}$ + Toluol; digeriert bei $37^{\circ} \mathrm{C} 24$ Stunden lang. Umpufferung und Ureasewirkung bei $\mathrm{pH} 7,0(1$ Stunde). Ammoniakbestimmung nach Folin. Die Angabe korrigiert wie in Tabelle V. .

\begin{tabular}{l|c|c|c|c}
\hline \multirow{2}{*}{ Enzymlösung } & \multicolumn{3}{|c}{ Zunahme des Säureverbrauchs } \\
\cline { 2 - 5 } & ohne $\mathrm{MnSO}_{4}$ & \multicolumn{2}{|c}{ mit $\mathrm{MnSO}_{4}$} \\
\hline $\begin{array}{c}\mathrm{ccm} \\
\mathrm{n}-\mathrm{H}_{2} \mathrm{SO}_{4}\end{array}$ & $\%$ & $0,02 \mathrm{n}-\mathrm{H}_{2} \mathrm{SO}_{4}$ & $\%$ \\
\hline Nierengewebe & 18,15 & 38,8 & 33,35 & 71,3 \\
Hypernephrom & 4,35 & 9,3 & 12,35 & 26,4
\end{tabular}

\section{Tabelle VII.}

Asparaginasewirkung des Hypernephroms und des Nierengevebes des Menschen.

Enzym: Ebenso wie in Tabelle III u. IV. Die Asparaginasebestimmung wurde in der Weise ausgeführt, wie in der Arbeit von Hiwatasi $(1941)^{12)}$ an- 
gegeben ist, und der Säureverbrauch zur Neutralisation des nach Foli in destillierten Ammoniaks in ccm 0,02 n-Schwefelsäure angegeben.

Versuchsansatz: $1 \mathrm{ccm} \mathrm{0,1} \mathrm{Mol-Asparaginlösung}+2 \mathrm{ccm}$ Gewebemazeration $+10 \mathrm{~cm}$ Phosphatpuffer $(\mathrm{pH} 7,5)+$ Toluol; digeriert bei $\mathrm{pH} 7,5$ und bei $37^{\circ} \mathrm{C} 24$ Stunden lang.

Kontrolle mit Enzym-Puffer ohne Asparagin. Abzug des Kontrollwertes. Die Tabellenangabe korrigiert.

Säureverbrauch

\begin{tabular}{c|c|c}
\hline Enzymlösung & ecm $0,02 \mathrm{n}-\mathrm{H}_{2} \mathrm{SO}_{4}$ & Spaltung \% \\
\hline Nierengewebe & 1,85 & $37 \%$ \\
\hline Hypernephrom & 0,50 & $10 \%$
\end{tabular}

\section{Analytischer Teil.}

\section{Tabelle VIIT.}

Stickstoffverteilung im wasserunlöslichen entfetteten Gewebe des Hypernephroms und des intakten Nierenteils.

Die Verteilungsanalyse des Hydrolysats des menschlichen Gewebes wurde nach der Vorschrift von Utzino u. Sh imazu $(1936)^{10)}$ und von Chen $(1939)^{13)}$ ãusgeführt.

\begin{tabular}{|c|c|c|c|c|c|c|}
\hline \multirow[b]{2}{*}{ N-Fraktion } & \multicolumn{3}{|c|}{ Hypernephrom } & \multicolumn{3}{|c|}{ Nierengewebe } \\
\hline & $\begin{array}{l}\mathrm{N} \mathrm{mg} \text { in } \\
2,0881 \mathrm{~g}\end{array}$ & $\%$ & $\begin{array}{c}\% \text { in } \\
\text { Gesamt-N }\end{array}$ & $\begin{array}{l}\mathrm{N} \text { mg in } \\
1,9965 \mathrm{~g}\end{array}$ & $\%$ & $\begin{array}{c}\% \text { in } \\
\text { Gesamt-N }\end{array}$ \\
\hline Gesamt-N & 288,4 & 13,81 & 100,00 & 319,20 & 15,99 & 100,00 \\
\hline Ammoniak-N & 19,18 & 0,92 & 6,65 & 20,33 & 1,02 & 6,37 \\
\hline Humin-N & 6,96 & 0,83 & 2,41 & 5,23 & 0,26 & 1,64 \\
\hline $\begin{array}{l}\text { Monoamino- } \\
\text { säure-N }\end{array}$ & 188,18 & 9,01 & 65,25 & 197,50 & 9,89 & 61,87 \\
\hline Purin-N & 3,20 & 0,15 & 1,11 & 5,19 & 0,26 & 1,63 \\
\hline Histidin-N & 9,66 & 0,46 & 3,35 & 8,01 & 0,40 & 2,51 \\
\hline Arginin-N & 25,62 & 1,71 & 12,35 & 38,93 & 1,95 & 12,20 \\
\hline Lysin-N & 18,26 & 0,64 & 4,60 & 19,18 & 0,96 & 6,01 \\
\hline Summe & 276,06 & 13,22 & 95,72 & 294,37 & 14,74 & 92,23 \\
\hline
\end{tabular}

